\title{
Evolution de monooxygénases hépatiques en fonction de l'âge chez les ovins de race Lacaune
}

\author{
M Kaddouri, C Eeckoutte, P Galtier \\ Laboratoire de Pharmacologie Toxicologie, INRA, 180, chemin de Tournefeuille, \\ $B P$ 3, 31931 Toulouse, France
}

Introduction - Le but du travail consiste à apprécier l'ontogenèse d'enzymes microsomales hépatiques d'oxydation chez les ovins femelles de race Lacaune.

Matériel et Méthodes - Sept lots de 6 brebis Lacaune provenant d'un même élevage, ont été utilisés; il s'agissait de fœetus de $90 \mathrm{j}$ et d'ovins de 1 et 4 semaines; $3,7,11$ mois et 6 ans (réforme). Le foie prélevé après saignée des animaux était divisé et les microsomes hépatiques préparés par ultracentrifugation. Les activités enzymatiques mesurées correspondaient aux dosages des cytochromes P-450 et $b 5$, cytochrome P-450 réductase, aminopyrine et benzphétamine $\mathrm{N}$-déméthylases; benzo$(\alpha)$ pyrène hydroxylase et 7-éthoxycoumarine-O déthylase. L'isoenzyme P-450 IIIA était quantifíée par électrophorèse suivie de Western blotting (Galtier et al, 1989).

Résultats et Discussion - Au cours de la première année, les protéines microsomales et le cytochrome P-450 total augmentaient régulièrement avec l'âge des animaux. Le cytochrome P-450 total non détectable chez le fœus au deuxième tiers de gestation (3,5 mois), était présent dès la première semaine d'âge post-natal $(0,40 \mathrm{nmol} / \mathrm{mg})$ puis augmentait chez les animaux d'un mois $(0,66 \mathrm{nmol} / \mathrm{mg})$ pour atteindre la valeur adulte au moment de la première gestation $(0,99 \mathrm{nmol} / \mathrm{mg})$. L'évolution de l'isoenzyme P-450 IIIA ne présen- tait aucune corrélation notable avec celle du cytochrome P-450 total ou l'âge des animaux; ainsi, l'agnelle d'une semaine présentait déjà une concentration comparable à celle de l'adulte $(0,31$ et 0,33 $\mathrm{nmol} / \mathrm{mg}$ ). Par ailleurs, l'évolution du cytochrome P-450 total était corrélée à celle de monooxygénases. La benzo $(\alpha)$ pyrène hydroxylase et l'aminopyrine $N$ déméthylase progressaient régulièrement du stade fotal à l'adulte de 6 ans (4 à 166 $\mathrm{pmol} / \mathrm{mg} / \mathrm{min}$ et 0.3 à $5.7 \mathrm{nmol} / \mathrm{mg} / \mathrm{min}$, respectivement). Par contre, la 7éthoxycoumarine Odééthylase non mesurable chez le fœetus était de $0,36 \mathrm{nmol} /$ $\mathrm{mg} / \mathrm{min}$ à 1 semaine et évoluait peu ensuite. Enfin, la benzphétamine $N$ déméthylase présente chez le fœtus $(0,15$ $\mathrm{nmol} / \mathrm{mg} / \mathrm{min})$ restait constante $(1,47$ à $1,93 \mathrm{nmol} / \mathrm{mg} / \mathrm{min}$ ) à partir de la première semaine de vie.

La connaissance de l'ontogenèse des monooxygénases ovines nous permet de mieux connaître les aptitudes des animaux à la métabolisation des nombreux dérivés endogènes, médicaments et toxiques dont la biotransformation dépend de l'activité des cytochromes P-450 hépatiques.

Galtier P, Paulin F, Eeckhoutte C, Larrieu G (1989) Food Chem Toxicol 27, 215-220 